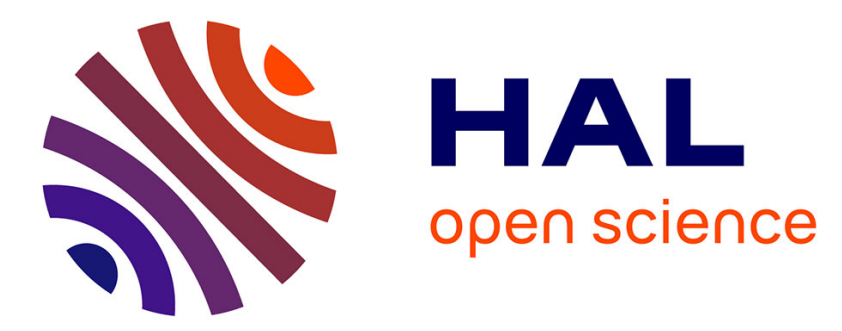

\title{
Synthetic conventional reflectometry probing of edge and scrape-o layer plasma turbulence
}

José Vicente, Filipe da Silva, T. Ribeiro, Stéphane Heuraux, Garrard D Conway, Bruce Scott, Luis Guimarãis, L. Gil, Egor Seliunin, P. Manz, et al.

\section{- To cite this version:}

José Vicente, Filipe da Silva, T. Ribeiro, Stéphane Heuraux, Garrard D Conway, et al.. Synthetic conventional reflectometry probing of edge and scrape-o layer plasma turbulence. Journal of Instrumentation, 2019, 14 (10), pp.C10043. 10.1088/1748-0221/14/10/C10043 . hal-02440064

\section{HAL Id: hal-02440064 \\ https://hal.univ-lorraine.fr/hal-02440064}

Submitted on 14 Jan 2020

HAL is a multi-disciplinary open access archive for the deposit and dissemination of scientific research documents, whether they are published or not. The documents may come from teaching and research institutions in France or abroad, or from public or private research centers.
L'archive ouverte pluridisciplinaire HAL, est destinée au dépôt et à la diffusion de documents scientifiques de niveau recherche, publiés ou non, émanant des établissements d'enseignement et de recherche français ou étrangers, des laboratoires publics ou privés. 


\section{Synthetic conventional reflectometry probing of edge and scrape-off layer plasma turbulence}

J. Vicente, ${ }^{a}{ }^{1}$ F. Da Silva, ${ }^{a}$ T. Ribeiro, ${ }^{b}$ S. Heuraux,${ }^{c}$ G.D. Conway, ${ }^{b}$ B. Scott, ${ }^{b}$ L. Guimarãis, ${ }^{a}$ L. Gil, ${ }^{a}$ E. Seliunin, ${ }^{a}$ P. Manz, ${ }^{b}$ J. Santos, ${ }^{a}$ A. Silva, ${ }^{a}$ C. Silva ${ }^{a}$ and the ASDEX Upgrade Team

${ }^{a}$ Instituto de Plasmas e Fusão Nuclear, Instituto Superior Técnico, Universidade de Lisboa, 1049-001 Lisboa, Portugal

${ }^{b}$ Max-Planck-Institut für Plasmaphysik, 85748 Garching, Germany

${ }^{c}$ Institut Jean Lamour, CNRS-University of Lorraine, BP 50840, 54011 Nancy Cedex, France

E-mail: jvicente@ipfn.tecnico.ulisboa.pt

ABSTRACT: Microwave reflectometry techniques have been successfully applied to measure electron density fluctuations in magnetic confined fusion devices with good spatial and time resolutions. However, quantitative interpretation of turbulence measurements has driven continuous development of both analytical theory and sophisticated numerical codes in support of reflectometry. Comparisons between experimental and synthetic reflectometry have been performed previously while only recently realistic gyro-fluid simulations have been employed together with a full-wave code to simulate measured turbulence properties with reflectometry. In this work, we report on recent efforts to employ the two-dimensional full-wave code REFMUL to implement a synthetic reflectometry diagnostic to model plasma turbulence measurements on the edge and scrape-off layer (SOL) peripheral regions of fusion devices. Numerical descriptions of microscopic turbulence were obtained from both an analytical model (following a Kolgomorov-like wavenumber spectrum) and from the GEMR turbulence code based on gyro-fluid theory. Simulations of fixed frequency conventional reflectometry with ordinary mode (O-mode) wave propagation were carried out for both turbulence cases separately. Preliminary comparisons between synthetic measurements, numerical plasma characteristics, and experimental data from ASDEX Upgrade tokamak are made. As previously described in literature, regimes of linear and non-linear response occurring at low and high turbulence levels, respectively, are observed and characterized. Synthetic spectra across moderate to high turbulence levels display qualitatively good agreement with experimental data across the edge region. KeYwords: Nuclear instruments and methods for hot plasma diagnostics; Simulation methods and programs

${ }^{1}$ Corresponding author. 


\section{Contents}

1 Introduction 1

2 Kolgomorov-like turbulence simulations 2

3 GEMR turbulence simulations $\quad 4$

4 ASDEX Upgrade turbulence measurements 4

5 Summary and discussion $\quad 6$

\section{Introduction}

Microwave reflectometry is a well-established technique that is employed for diagnostic of several parameters in nuclear fusion devices (e.g. see [1] for an overview). The high spatial and temporal resolutions of reflectometer measurements allow investigating phenomena, such as plasma turbulence, critical for the performance of fusion machines. Nevertheless, the complex interaction between probing electromagnetic waves and the turbulent plasma medium has required a strong modelling effort in support of reflectometry. This has driven continuous development of both analytical theories and numerical simulations in order to obtain quantitative measurements from the experimental observations. Measurements obtained at the edge and scrape-off layer (SOL) regions of tokamaks are particularly challenging because of high turbulence levels and high density particle bursts observed in these regions during L-mode plasma discharges. Measurements of ion-saturation current, which are a proxy for electron density, at the ASDEX Upgrade (AUG) tokamak display fluctuation levels ranging from $\sim 10 \%$ inside the Last Closed Flux Surface (LCFS) to $\sim 40 \%$ in the SOL [2]. Additionally, coherent plasma structures known as blobs or filaments are usually measured in the far-SOL with relative density amplitude of the order of 100\% [3].

In this work, we report on recent results obtained with a synthetic reflectometry diagnostic based on REFMUL, a two-dimensional full-wave code for ordinary mode (O-mode) wave propagation [4]. In O-mode propagation the cut-off condition sets the critical electron density $n_{c}$ for reflection to occur in the plasma, where $n_{c}=f_{\mathrm{o}}^{2}\left(4 \pi^{2} \varepsilon_{\mathrm{o}} m_{\mathrm{e}} / e^{2}\right)$ with $f_{\mathrm{o}}$ being the probing frequency, $e$ and $m_{\mathrm{e}}$ the electron charge and mass, and $\varepsilon_{0}$ the permittivity of free space. REFMUL simulations are carried out on a Cartesian grid with spatial discretization $\delta x=\delta y=3.75 \times 10^{-4} \mathrm{~m}$ corresponding to a small fraction of the probing wavelength. The time discretization is also a small fraction of the wave period in order to comply with the Courant-Friedrichs-Lewy (CFL) condition to ensure numerical stability. The case of fixed frequency probing in conventional set-up (i.e. normal incidence) is treated here to investigate synthetic measurements of turbulence in the periphery of a medium sized tokamak. This kind of systems have been traditionally devoted to study density fluctuations. 
The AUG tokamak [5] is equipped with several reflectometry systems and was considered as experimental reference. An example of O-mode fixed frequency reflectometers in conventional set-up at AUG can be found in [6]. The characteristics of these particular systems, namely the monostatic set-up, the beam width, probing frequencies and In-phase/Quadrature detection scheme, were also considered when implementing the synthetic diagnostic used here. A more detailed description of the synthetic diagnostic can be found elsewhere [7].

In previous work, an analytical construct to model broadband plasma turbulence has been employed [7]. In those cases, a Kolgomorov-like wavenumber spectrum was used and the turbulence level $\delta n_{\mathrm{e}} / n_{\mathrm{e}}$ was varied in a set of different simulations. In section 2 of this paper the results obtained with the same model, but for an extended range of turbulence levels, accounting for more realistic SOL values, are presented.

On the other hand, coupling of a numerical turbulence code with a reflectometry code has been achieved in the past (e.g. [8] where a Landau-fluid model was used). More recently, the GEMR code $[9,10]$ which is based on gyro-fluid theory and able to provide improved numerical descriptions of the microscopic properties of edge/SOL turbulence, has been coupled to REMUL [11]. A match between GEMR simulations and AUG experimental plasmas was envisaged by carrying out GEMR runs with AUG-like plasma parameters and performing AUG plasma discharges with circular magnetic geometry since GEMR is not able to simulate the X-point geometry of usual AUG divertor configurations. In section 3, results from REFMUL/GEMR simulations are presented and finally in section 4 a preliminary comparison between synthetic data and experimental data is carried out.

\section{Kolgomorov-like turbulence simulations}

Simulations with fixed probing frequency $f_{\mathrm{o}}=49 \mathrm{GHz}$ have been carried using two different electron density gradient lengths $L_{n}$, corresponding to cases of typical shallow $\left(L_{n}=16 \mathrm{~cm}\right)$ and steep $\left(L_{n}=5 \mathrm{~cm}\right)$ background density profiles that can be observed at the edge of L-mode plasmas at AUG. Density fluctuations with a 2D Kolgomorov-like wavenumber spectrum, i.e. a flat spectrum at low k-values followed by a power law decay above a given "knee" position $\left(k_{\text {knee }}=4 \mathrm{~cm}^{-1}\right.$ in these cases) were superposed to slab background plasmas. The root mean square of the density fluctuations at the cut-off position (i.e. turbulence level at the cut-off $\delta n_{\mathrm{e}} / n_{c}$ ) was varied on a simulation-to-simulation basis, covering the range of $\sim 0.02-70 \%$. A radial turbulence flow was also imposed with constant velocity $v_{\text {siml }}=0.013 c$ where $c$ is the velocity of light.

The reflectometry phase fluctuations registered along each turbulence level in both background density gradient cases are displayed in figure 1 .

A linear relation between the phase fluctuations and the turbulence levels, at low $\delta n_{\mathrm{e}} / n_{c}$ values, is observed as in previous studies [7]. A transition to non-linear regime is observed at moderate 2-4\% $\delta n_{\mathrm{e}} / n_{c}$ values which is followed by saturation where increasing turbulence levels no longer increase significantly the reflectometer phase fluctuations. The linear regime is in excellent agreement with simplified 1D theory that accounts for Bragg backscattering (BBS) as the main scattering mechanism. To illustrate this, the theoretical estimates of phase fluctuations for the case of small

coherent perturbations of amplitude $\delta n$, provided by $\delta \varphi=\sqrt{\pi} k_{\mathrm{o}}\left(L_{n} / k_{f}\right)^{1 / 2} \delta n / n_{c}$ (see ref. [12]), were computed using an effective wavenumber component $k_{f}=3 \mathrm{~cm}^{-1}$ of the density fluctuation 


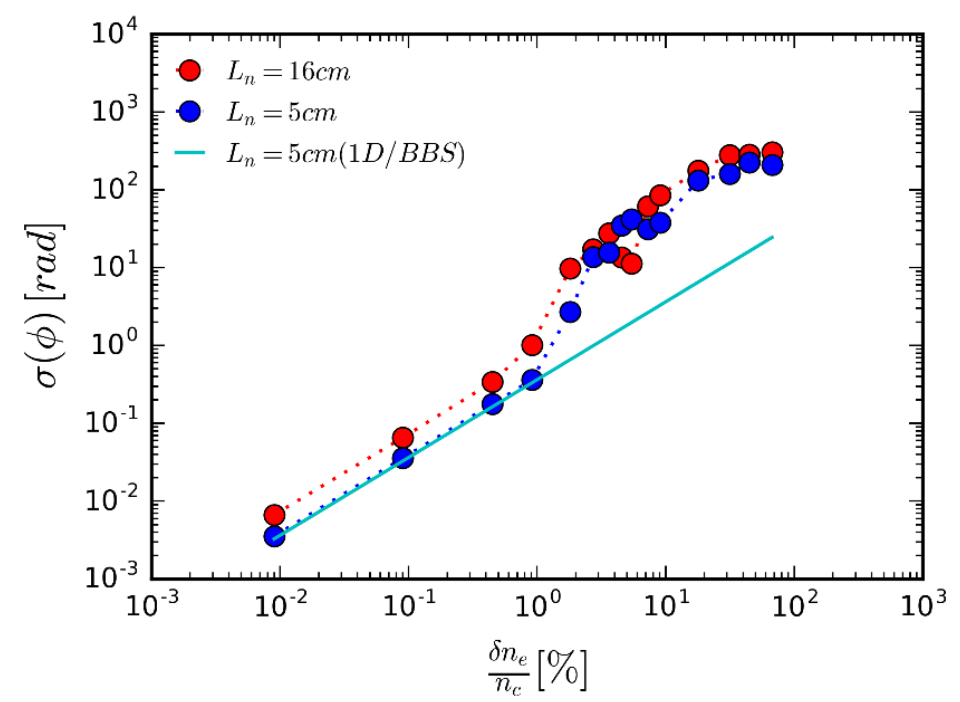

Figure 1. Standard deviation of simulated reflectometry phase $\varphi(t)$ signals against the corresponding turbulence levels at the cut-off location. Also shown (solid line) is the theoretical estimate of 1D Bragg backscattering contributions, given in [12] for the case of $L_{n}=5 \mathrm{~cm}$, with $k_{f}=3 \mathrm{~cm}^{-1}$.

spectrum and are displayed in figure 1 together with the simulated data. Note that the $k_{f}$ value was obtained by fitting the 1D theoretical estimate to the data in the linear region.

The behavior of the corresponding reflectometry amplitude signals along the turbulence level scans is shown in figure 2 where both the average and the fluctuating values are displayed.
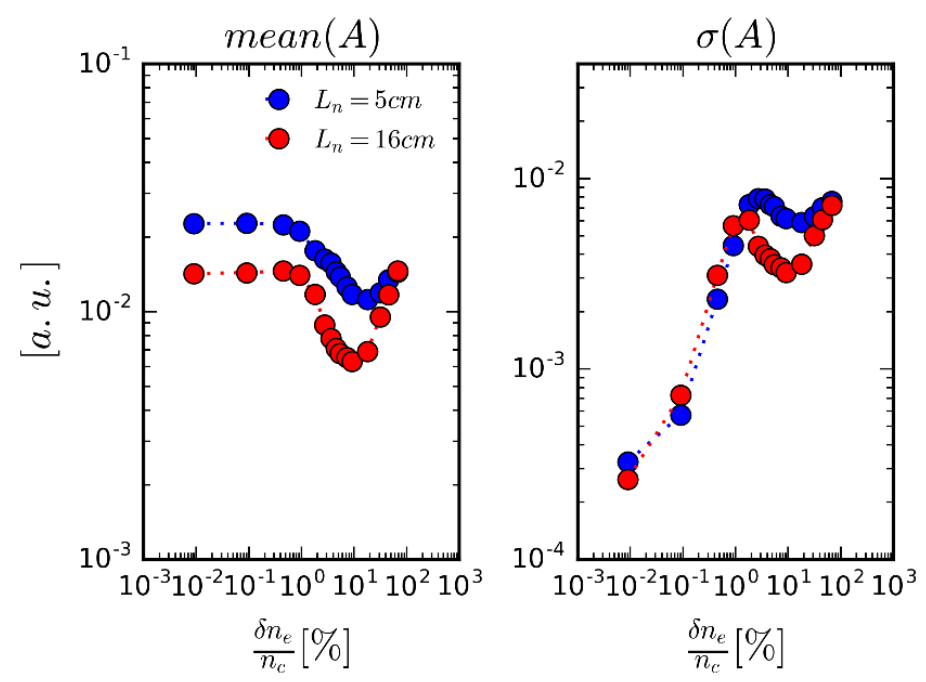

Figure 2. Average and standard deviation of simulated reflectometry amplitude $A(t)$ signals against the corresponding turbulence levels at the cut-off location.

The features observed in the amplitude signals highlight the importance of $2 \mathrm{D}$ effects at moderate and high turbulence levels. The drop in average amplitude with increasing gradient 
length must be due to geometrical effects given the larger beam spreading associated with longer plasma paths. These are competing with BBS efficiency and result in small angle multi-scattering leading up to the non-linear regime [13]. With increasing turbulence levels, the amplitude drops and at a given point rises again. The latter is possibly due to enhanced BBS in a region closer to the antenna, ahead of the cut-off location [14]. This should be further investigated in future work.

\section{GEMR turbulence simulations}

GEMR is a code based on a 3D electromagnetic gyro-fluid model with global geometry, in the sense that both poloidal and radial dependencies are considered without using the flux tube approximation. It solves the first six moments of the gyro-kinetic equation computing the evolution of density fluctuations and density profile gradient. GEMR density data displays a narrow turbulence level profile, in the vicinity of the LCFS, and at lower values than expected in experiments [11]. However, the self-consistent evolution of GEMR turbulence is closer to reality, in the sense that the turbulence character and dynamics across the edge and SOL are well reproduced [15]. Coupling of GEMR with REFMUL has been reported recently, see details and first results in [11]. Here, the original turbulent density output data of GEMR was taken and a scaling factor was applied in order to simulate the reflectometer diagnostic on a larger range of turbulence levels. The electron density data was rendered on a poloidal plane after post processing the built-in GEMR field aligned coordinates. Hence, the cross-section of the magnetic flux surfaces was taken into account. The equilibrium density profiles, which are evolved along the GEMR simulation, determine the O-mode frequencies that can probe the region of interest. In this case, the edge and SOL were accessible in the range 28$32 \mathrm{GHz}$. To illustrate the reflectometer response to the turbulence level, the probing wave frequency $f_{\mathrm{o}}=30 \mathrm{GHz}$ was employed, for which the density gradient length, at the corresponding cut-off location, was of $L_{\mathrm{n}}=10 \mathrm{~cm}$. The power spectra of the complex amplitude signals are displayed in figure 3 together with the corresponding integrated power. A strong spectral broadening effect with increasing turbulence levels is observed, in particular up to $\delta n_{\mathrm{e}} / n_{\mathrm{e}} \sim 20 \%$. Similarly, the total integrated spectral power scales linearly with $\delta n_{\mathrm{e}} / n_{\mathrm{e}}$ up to a given turbulence level (10-20\%) above which it saturates.

\section{ASDEX Upgrade turbulence measurements}

A set of deuterium plasma discharges have been performed at AUG where L-mode scenarios were obtained using so called inner limiter configurations to establish circular magnetic geometries, as in GEMR simulations. AUG discharge \#35164 is considered here where measurements were made with plasma current $I_{p}=0.63 \mathrm{MA}$, toroidal magnetic field $B_{t}=-2.2 \mathrm{~T}$ (ion drift direction towards the X-point near the lower divertor), safety factor at the flux surface enclosing $95 \%$ of the total poloidal flux $q_{95}=4.0$, line integrated core plasma density $\bar{n}_{\mathrm{e}}=4.3 \times 10^{19} \mathrm{~m}^{-3}$ and auxiliary electron cyclotron resonance heating $P_{\mathrm{ECRH}}=0.6 \mathrm{MW}$.

An O-mode reflectometer considered here, operating in the microwave band Q (33.0-49.2 GHz), located at the low magnetic field side mid-plane, has frequency hopping capability [6]. The data acquisition rate of the reflectometry hopping systems is currently $2 \mathrm{MHz}$. The reflectometer was set to operate at 6 different frequency steps $(33.0,40.0,43.0,44.9,46.7$ and $48.3 \mathrm{GHz})$ during $25 \mathrm{~ms}$ 

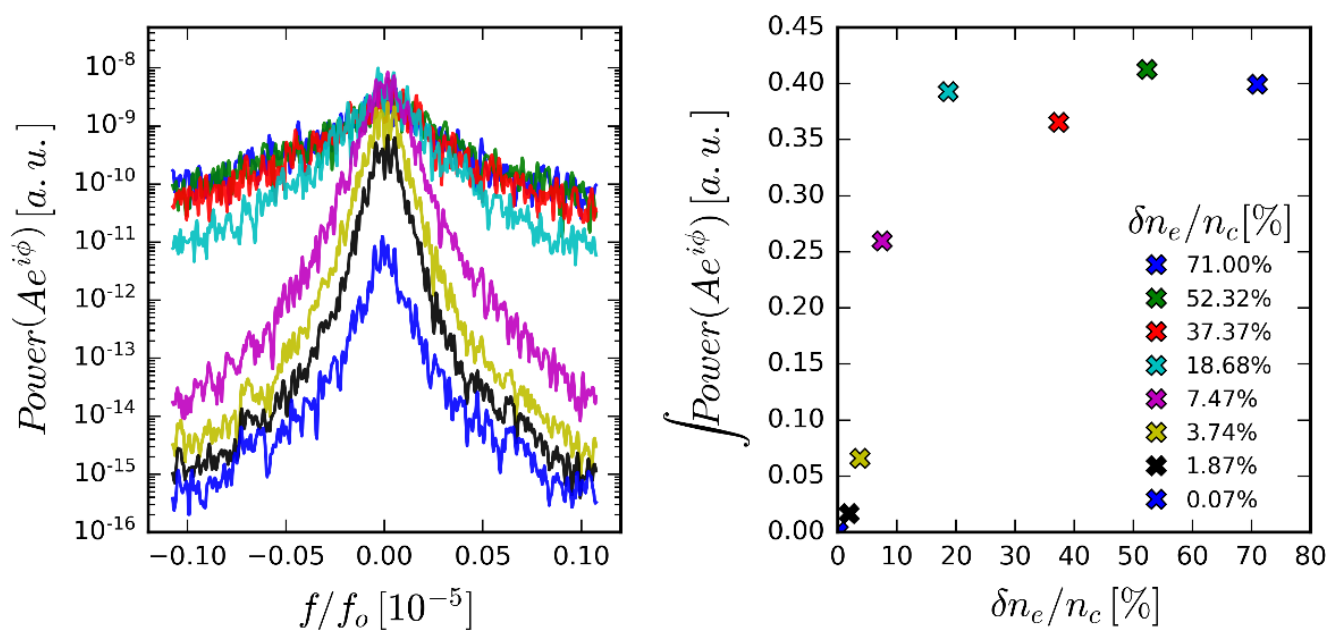

Figure 3. Left: power spectra of the reflectometry complex amplitude signals $A e^{i \varphi}$ obtained from simulations with fixed probing frequency $f_{\mathrm{o}}=30 \mathrm{GHz}$ over a turbulence level scan of GEMR data. Right: total integrated power of the corresponding spectra on the left panel, against the turbulence level.
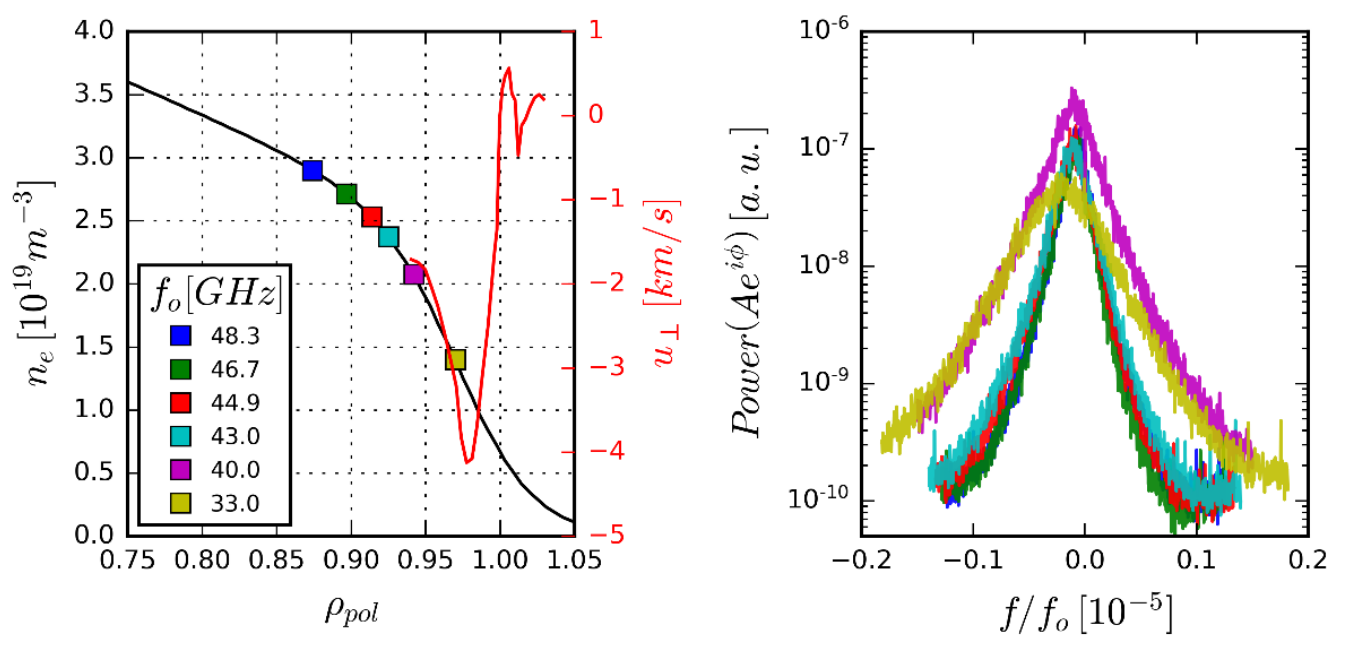

Figure 4. Left: electron density profile, fitted to data obtained with Thomson Scattering and Li-beam diagnostics, and perpendicular velocity profile, obtained from a Doppler reflectometry system, for AUG discharge \#35164, $t=2.5 \mathrm{~s}$. Also shown are the cut-off positions at the density profile corresponding to a set of O-mode probing frequencies. Right: power spectra of the experimental reflectometry complex amplitude signals $A e^{i \varphi}$ obtained with the corresponding probing frequencies in the left panel.

each. In figure 4 it is displayed an electron density profile (fit to data from Thomson Scattering and Li-beam diagnostics) where the cut-off layers of the corresponding probing frequencies are indicated. Also displayed in the figure is the perpendicular velocity profile obtained from an Xmode Doppler system [16]. It is observed that the probed region lies in the edge, inside a velocity shear layer, while the minimum probing frequency is nevertheless too high to probe the SOL. 
The spectra of the experimental complex amplitude signals obtained from the Q-band reflectometer are also displayed in figure 4, in the right panel. A strong spectral broadening is observed, in particular for the two outermost probed layers, which also display small Doppler shifts.

A qualitative comparison between the spectra in figure 3 and in figure 4 shows that the shape of GEMR spectra at moderate turbulence levels compares well with AUG spectra at inner locations, and that the GEMR spectra at the highest turbulence level displays very enhanced broadening similarly to what AUG spectra displays at the furthest out locations, where higher turbulence levels are indeed expected. Along with turbulence level dependency, an impact of the poloidal propagation of turbulence on the spectral broadening should not be excluded. This should be further investigated together with the possibility to recover the wavenumber spectra and in particular the spectral decay index.

\section{Summary and discussion}

Reflectometry measurements of plasma density fluctuations in the edge and SOL regions have been modelled using a synthetic diagnostic. To obtain the numerical description of the plasma, two different models were used: one aiming to reproduce a Kolgomorov-like wavenumber spectra and the other a gyro-fluid model included in the GEMR code. The former was previously studied in some detail in ref. [6] and the latter was employed just recently with first results submitted in ref. [10]. In those previous studies, the maximum density fluctuation levels were not representative of the whole edge and SOL regions. In this work, density fluctuation levels have been extended up to $\sim 70 \%$ to cover the high $\delta n_{\mathrm{e}} / n_{\mathrm{e}}$ values usually found in the plasma periphery. In the case of Kolgomorov-like turbulence, simulations show that in the $\delta n_{\mathrm{e}} / n_{\mathrm{e}}$ range of $4-30 \%$ there is a strong non-linear regime, above which a saturation in the phase response occurs. On the other hand, results of the turbulence level scan using the GEMR data show spectral broadening of the complex amplitude signals with increasing turbulence levels as well as the total integrated spectral power scaling linearly with the turbulence levels up to $\delta n_{\mathrm{e}} / n_{\mathrm{e}} \sim 10-20 \%$. If GEMR data is more realistic than the Kolgomorov model, as expected, the experimental measurements should remain in the linear regime at slightly higher $\delta n_{\mathrm{e}} / n_{\mathrm{e}}$ values. A first comparison between experimental and simulation data has been performed showing reasonable qualitative agreement and demonstrating experimental sensitivity in regions where turbulence levels are expected to be higher. Further analysis and studies are underway to include simultaneous experimental measurements with Langmuir probes and use of numerical plasma models that include profile shapes similar to those obtained experimentally. Nevertheless, implementation of synthetic reflectometry diagnostics with realistic turbulence models, as displayed here, promise to enhance and further support the reflectometry measuring capabilities. On the other hand, a fully realistic description of the wave propagation in turbulent plasmas may require employing 3D modelling. In particular, to properly describe the behaviour of electric fields in large 3D cavities. REFMUL3, a three-dimensional full-wave code enabled with parallel computing, was recently developed and benchmarked against the 2D code REFMUL [17]. First results displayed robust phase reconstruction, although obtained without plasma turbulence, which strengthens our confidence in 2D simulations with this respect. While 3D simulations are still computationally expensive and a scarce resource they should be used in the near future to enlighten the possibilities of conventional reflectometry measurements (e.g. regarding turbulence levels and wavenumber spectra) under the challenging edge and SOL plasma turbulence conditions. 


\section{Acknowledgments}

This work has been carried out within the framework of the EUROfusion Consortium and of the French Federation for Magnetic Fusion Studies (FR-FCM) and has received funding from the Euratom research and training programme 2014-2018 and 2019-2020 under grant agreement No 633053. The views and opinions expressed herein do not necessarily reflect those of the European Commission. IST activities also received financial support from "Fundação para a Ciência e Tecnologia" through project UID/FIS/50010/2019.

\section{References}

[1] T. Estrada et al., Microwave reflectometry diagnostics: Present day systems and challenges for future devices, Plasma Fus. Res. 7 (2012) 2502055.

[2] B. Nold et al., Generation of blobs and holes in the edge of the ASDEX Upgrade tokamak, Plasma Phys. Control. Fusion 52 (2010) 065005.

[3] G. Birkenmeier et al., Filament transport, warm ions and erosion in ASDEX Upgrade L-modes, Nucl. Fusion 55 (2015) 033018.

[4] F. da Silva et al., Unidirectional transparent signal injection in finite-difference time-domain electromagnetic codes -application to reflectometry simulations, J. Comput. Phys. 203 (2005) 467.

[5] A. Kallenbach et al., Overview of ASDEX Upgrade results, Nucl. Fusion 57 (2017) 102015.

[6] L. Cupido et al., Frequency hopping millimeter-wave reflectometry in ASDEX upgrade, Rev. Sci. Instrum. 77 (2006) 10E915.

[7] J. Vicente et al., Turbulence level effects on conventional reflectometry using 2D full-wave simulations, Rev. Sci. Instrum. 89 (2018) 10H110.

[8] G.D. Conway et al., Coupling of turbulence and reflectometer simulation codes and comparison with experiment, Plasma Phys. Control. Fusion 44 (2002) 451.

[9] B.D. Scott, Free-energy conservation in local gyrofluid models, Phys. Plasmas 12 (2005) 102307.

[10] S.J. Zweben et al., Comparison of scrape-off layer turbulence in Alcator C-Mod with three dimensional gyrofluid computations, Phys. Plasmas 16 (2009) 082505

[11] J. Vicente et al., 2D full-wave simulations of conventional reflectometry using 3D gyro-fluid plasma turbulence, submitted to Plasma Phys. Control. Fusion.

[12] C. Fanack et al., Ordinary-mode reflectometry: modification of the scattering and cut-off responses due to the shape of localized density fluctuations, Plasma Phys. Control. Fusion 38 (1996) 1915.

[13] E.Z. Gusakov et al., Non-linear theory of fluctuation reflectometry, Plasma Phys. Control. Fusion 44 (2002) 1565.

[14] E.Z. Gusakov et al., Strong Bragg backscattering in reflectometry, Plasma Phys. Control. Fusion 51 (2009) 065018.

[15] T.T. Ribeiro et al., Gyrofluid turbulence studies of the effect of the poloidal position of an axisymmetric Debye sheath, Plasma Phys. Control. Fusion 50 (2008) 055007.

[16] G.D. Conway et al., Plasma rotation profile measurements using Doppler reflectometry, Plasma Phys. Control. Fusion 46 (2004) 951.

[17] F. da Silva et al., Benchmarking 2D against 3D FDTD codes in the assessment of reflectometry performance in fusion devices, 2019 JINST 14 C08004. 